\title{
KUROSH'S CHAINS OF ASSOCIATIVE RINGS
}

\author{
by R. R. ANDRUSZKIEWICZ and E. R. PUCZYŁOWSKI
}

(Received 1 October, 1988)

Let $N$ be a homomorphically closed class of associative rings. Put $N_{1}=N^{1}=N$ and, for ordinals $\alpha \geqslant 2$, define $N_{\alpha}\left(N^{\alpha}\right)$ to be the class of all associative rings $R$ such that every non-zero homomorphic image of $R$ contains a non-zero ideal (left ideal) in $N_{\beta}$ for some $\beta<\alpha$. In this way we obtain a chain $\left\{N_{\alpha}\right\}\left(\left\{N^{\alpha}\right\}\right)$, the union of which is equal to the lower radical class $I N$ (lower left strong radical class $l s N$ ) determined by $N$. The chain $\left\{N_{\alpha}\right\}$ is called Kurosh's chain of $N$. Suliński, Anderson and Divinsky proved [7] that $l N=\bigcup_{i=1}^{\infty} N_{i}$. Heinicke [3] constructed an example of $N$ for which $l N \neq N_{k}$ for $k=1,2, \ldots$ In [1] Beidar solved the main problem in the area showing that for every natural number $n \geqslant 1$ there exists a class $N$ such that $l N=N_{n+1} \neq N_{n}$. Some results concerning the termination of the chain $\left\{N^{\alpha}\right\}$ were obtained in $[2,4]$. In this paper we present some classes $N$ with $N_{\alpha}=N^{\alpha}$ for all $\alpha$. Using this and Beidar's example we prove that for every natural number $n \geqslant 1$ there exists an $N$ such that $N_{\alpha}=N^{\alpha}$ for all $\alpha$ and $N_{n} \neq N_{n+1}=N_{n+2}$. This in particular answers Question 6 of [4].

All rings in the paper are associative and $N$ is a homomorphically closed class of such rings. To denote that $I$ is an ideal (left ideal) of a ring $R$ we write $I \triangleleft R(I<R)$. A subring $A$ of a ring $R$ is called accessible (left accessible) if there is a chain of subrings $A=A_{n} \subseteq A_{n-1} \subseteq \ldots \subseteq A_{0}=R$ such that $A_{i} \triangleleft A_{i-1}\left(A_{i}<A_{i-1}\right)$ for $i=1,2, \ldots, n$.

$\mathbb{Z}$ is used to denote the ring of integers, $\mathbb{Q}$ the field of rational numbers and $\mathbb{Q}(i)$ the field of complex numbers $a+b i$, where $a, b \in \mathbb{Q}$.

The fundamental definitions and properties of radicals may be found in [8] and those of strong radicals in [2]. The following proposition collects some well known properties of classes $N_{\alpha}$ and $N^{\alpha}$.

Proposition 1. (i) $N_{\alpha} \subseteq N^{\alpha}$ for every ordinal $\alpha$;

(ii) classes $N_{\alpha}$ and $N^{\alpha}$ are homomorphically closed for all $\alpha$;

(iii) ([2]) if $0 \neq R \in l s N$ then $R$ contains a non-zero left accessible subring in $N$;

(iv) ([7]) $R \in I N$ if and only if every non-zero homomorphic image of $R$ contains a non-zero accessible subring in $N$;

(v) ([2]) if $0 \neq R \in N^{n+1}$, where $n$ is an integer $\geqslant 1$, then there are subrings $0 \neq L_{n}<\ldots<L_{0}=R$ of $R$ such that $L_{n} \in N$;

(vi) [7] $R \in N_{n+1}$, where $n$ is an integer $\geqslant 1$, if and only if every non-zero homomorphic image $R^{\prime}$ of $R$ contains subrings $0 \neq I_{n} \triangleleft \ldots \triangleleft I_{0}=R^{\prime}$ such that $I_{n} \in N$.

Recall that a radical class $S$ is called left stable if for every $L<R, S(L) \subseteq S(R)$. An example of a left stable radical class is the generalized nil radical $N_{g}$; this is the upper radical determined by the class of reduced rings i.e. rings without non-zero nilpotent elements.

THEOREM 1. If $S$ is a left stable radical class containing $N_{g}$ then for $N=S \cup P$, where $P$ is a homomorphically closed class of commutative rings, $N_{\alpha}=N^{\alpha}$ for every ordinal $\alpha$.

Glasgow Math. J. 32 (1990) 67-69. 
Proof. In view of Proposition 1 it suffices to prove that if $0 \neq L_{n}<\ldots<L_{0}=R$ and $L_{n} \in N$ then there are $0 \neq I_{n} \triangleleft \ldots \triangleleft I_{0}=R$ with $I_{n} \in N$. It is so if $S(R) \neq 0$. Hence, since $N_{g} \subseteq S$ and $S$ is left stable, we can assume that the ring $R$ is reduced and $L_{n} \in P$. Let $k$ be a minimal number such that $L_{n}$ is contained in the centre of $L_{k}$. Suppose that $k \geqslant 1$. Then there are $l \in L_{n}$ and $l^{\prime} \in L_{k-1}$ such that $l l^{\prime}-l^{\prime} l \neq 0$. Now $\left(l l^{\prime}-l^{\prime} l\right)^{2}=\left(l l^{\prime}\right)^{2}-l\left(l^{\prime}\right)^{2} l-$ $l^{\prime} l^{2} l^{\prime}+\left(l^{\prime} l\right)^{2}$. Since $l^{\prime} l,\left(l^{\prime}\right)^{2} l \in L_{k}$ and $L_{n}$ is contained in the centre of $L_{k}$, we have $\left(l l^{\prime}\right)^{2}=l^{\prime} l^{2} l^{\prime}, l\left(l^{\prime}\right)^{2} l=\left(l^{\prime}\right)^{2} l^{2}$ and $\left(l^{\prime} l\right)^{2}=\left(l^{\prime}\right)^{2} l^{2}$. Thus $\left(l l^{\prime}-l^{\prime} l\right)^{2}=0$ and, since the ring $R$ is reduced, $l l^{\prime}-l^{\prime} l=0$. This contradiction shows that $L_{n}$ is contained in the centre of $R$. On the other hand $R L_{n}^{n} \subseteq L_{n}$. Hence $L_{n}=L_{n}+R L_{n}^{n} \triangleleft L_{n}+R L_{n}^{n-1} \triangleleft \ldots \triangleleft L_{n}+R L_{n} \triangleleft R$ and the result follows.

Let $p$ be a prime of the form $4 m+3$ and, for $n \geqslant 0$, let $A_{n}$ be the subring of $\mathbb{Q}(i)$ generated by $p$ and $i p^{n}$. The following properties of the rings $A_{n}$ were established by Beidar in [1, Lemma 1].

Proposition 2. (i) $A_{n} \triangleleft A_{m}$ if and only if $n=m$ or $n=m+1$;

(ii) proper homomorphic images of $A_{n}$ are finite;

(iii) the only subring of $\mathbb{Q}(\mathrm{i})$ isomorphic to $A_{n}$ is the ring $A_{n}$ itself;

(iv) if $B$ is a subring of $\mathbb{Q}(\mathrm{i})$ and $A_{n} \triangleleft B$ then $1 \in B$ or $B=A_{n}$ or $B=A_{n-1}$.

Now we prove the following theorem.

THEOREM 2. If $N=N_{g} \cup T \cup\left\{A_{n}\right\}$, where $T$ is the class of rings whose additive groups are torsion and $\left\{A_{n}\right\}$ is the class of all isomorphic images of $A_{n}$ for an $n \geqslant 1$, then $N_{1}=N^{1} \subsetneq N_{2}=N^{2} \subsetneq \ldots \subsetneq N_{n+1}=N^{n+1}=N_{n+2}=N^{n+2}$.

Proof. As an immediate consequence of Theorem 1 and Proposition 2(ii) one obtains that, for every ordinal $\alpha, N_{\alpha}=N^{\alpha}$. It follows from Proposition 1 (vi) and Proposition 2 that $A_{0} \in N_{n+1} \backslash N_{n}$. Hence $N_{1} \subsetneq N_{2} \subsetneq \ldots \subsetneq N_{n+1}$. It remains to prove that $l N=N_{n+1}$ or, equivalently, that every non-zero ring $R \in I N$ contains a non-zero ideal in $N_{n}$. Obviously we can assume that $R$ is semiprime and the additive group of $R$ is torsion-free. Then by Propositions 1 (iv) and 2 (ii), $R$ contains an accessible subring isomorphic to $A_{n}$. Let $t$ be the minimal integer $\geqslant 0$ such that there are $I_{t} \triangleleft I_{t-1} \triangleleft \ldots \triangleleft I_{0}=R$ with $I_{t}$ isomorphic to $A_{k}$ for some $0<k \leqslant n$. We claim that $t \leqslant 1$. For, suppose that $t \geqslant 2$ and take $I$ the ideal of $I_{t-2}$ generated by $I_{t}$. By Andrunakievich's lemma, $I^{3} \subseteq I_{t}$. This, semiprimeness of $R$ and properties of $A_{n}$ imply that $I$ is a prime ring without 1 . Since the additive group of $I$ is torsion-free, we can form the quotient ring $\mathbb{Z}^{-1} I$. Now $\mathbb{Z}^{-1} I_{t} \triangleleft \mathbb{Z}^{-1} I$ and $\mathbb{Z}^{-1} I_{t}$, being isomorphic to $\mathbb{Q}(\mathrm{i})$, contains 1 . However the ring $\mathbb{Z}^{-1} I$ is prime, so $\mathbb{Z}^{-1} I_{t}=\mathbb{Z}^{-1} I$. Thus $I$ is a ring without 1 isomorphic to a subring of $\mathbb{Q}(\mathrm{i})$. By Proposition 2 (iii) and (iv), $I$ is isomorphic to $A_{n}$ or $A_{n-1}$. Moreover, if $I$ is isomorphic to $A_{n-1}$ then, since $I$ is a ring without $1, n-1>0$. This and the fact that the sequence $I \triangleleft I_{t-2} \triangleleft I_{t-3} \triangleleft \ldots \triangleleft I_{0}=R$ is shorter than $I_{t} \triangleleft I_{t-1} \triangleleft \ldots \triangleleft I_{0}=R$ prove the claim. Thus $R$ contains a non-zero ideal isomorphic to $A_{k}$ for some $0<k \leqslant n$. It is clear from Proposition 2 that $A_{k} \in N_{n}$. The result follows.

Remark. Let $N=N_{g} \cup T \cup\left\{A_{2} n: n=1,2, \ldots\right\}$. One can easily check using Propositions 1 and 2 that for every $0<i<2^{n}, n=1,2, \ldots, A_{2} n_{+i} \in N_{2} n_{-i+1} \backslash N_{2} n_{-i}$. Hence by Theorem $1, N_{k}=N^{k} \subsetneq N_{k+1}=N^{k+1}$ for $k=1,2, \ldots$ and $l N=l s N=\bigcup_{k=1}^{\infty} N^{k}$. 
In [4] it was proved that if the class $N$ is hereditary (hereditary and contains nilpotent rings) then $l s N=N^{4}\left(l s N=N^{3}\right)$ i.e. in those cases the chain $\left\{N^{\alpha}\right\}$ terminates one step further than $\left\{N_{\alpha}\right\}$. In [6] Stewart proved that if $N$ is a class of zero rings then $l N=N_{3}$. The following theorem shows in particular that in this case $l s N=l N=N^{3}$. It can also be regarded as a generalization of the fact that the prime radical is strong.

THEOREM 3. If $N$ is a class of zero rings then $S=l N$ is a left strong radical class.

Proof. Suppose that $L<R$ and $L \in S$. Let $U=\{x \in L: L x=0\}$. If $U=L$ then $L^{2}=0$ and it is easy to check that $L R^{1} \in S$, where $R^{1}$ is the ring $R$ with an unity adjoined. Suppose $U \neq L$. Then $0 \neq L / U \in S$, so $L / U$ contains a non-zero accessible subring $A / U \in N$. Hence there are subrings $A_{0}, \ldots, A_{n}$ of $L$ such that $A \triangleleft A_{n} \triangleleft \ldots \triangleleft A_{0}=L$. Now $0 \neq \mathrm{LAR}^{1} \triangleleft R$. Since $\left(\mathrm{LAR}^{1}\right)^{m} \subseteq(\mathrm{LA})^{m} R^{1}$ for $m=1,2, \ldots$ and the ideal of $L$ generated by $A$ is nilpotent, $\mathrm{LAR}^{1}$ is a nilpotent ideal of $R$. Suppose that $\left(\mathrm{LAR}^{1}\right)^{k+1}=0$, $\left(\mathrm{LAR}^{1}\right)^{k} \neq 0$ and take $t \in\left(\mathrm{LAR}^{1}\right)^{k-1}$. Obviously $\operatorname{LAR}^{1} t R^{1} \triangleleft R$. For every $l \in L, x, y \in R^{1}$, the map $f: A \rightarrow$ lAxty given by $f(a)=$ laxty is a ring epimorphism and lAxty $\triangleleft L A R^{1} t R^{1}$. Hence $\left(\mathrm{LAR}^{1}\right)^{k} \in S$. Therefore $S(R) \neq 0$ and the result follows.

Let us observe that if $N$ is a class of $N$-nilpotent rings [5] and $N_{0}$ is the class of zero $l N$-radical rings then $l N_{0}=l\left(N_{0} \cap N\right)$. Applying [5, Theorem 4] to $\alpha=l N$ one obtains that $R^{0} \in N$, where $R^{0}$ is the zero ring on the additive group of a ring $R \in N$. The same theorem applied to $\alpha=l N_{0}$ implies $N \subseteq l N_{0}$. Hence $l N=L N_{0}$ and Theorem 3 gives

COROLLARY. If $N$ is a class of $M$-nilpotent rings then $l N=l s N=N_{3}=N^{3}$.

\section{REFERENCES}

1. K. I. Beidar, A chain of Kurosh may have an arbitrary finite length, Czech. Math. J. 32 (1982), 418-422.

2. N. Divinsky, J. Krempa and A. Suliński, Strong radical properties of alternative and associative rings, J. Algebra 17 (1971), 369-381.

3. A. Heinicke, A note on lower radical constructions for associative rings, Canad. Math. Bull. 11 (1968), 23-30.

4. E. R. Puczyłowski, On questions concerning strong radicals of associative rings, Quaestiones Math. 10 (1987), 321-338.

5. A. D. Sands, On $M$-nilpotent rings, Proc. Royal Soc. Edinburgh Sect. A 93 (1982), 63-70. 31-32.

6. P. N. Stewart, On the lower radical construction, Acta Math. Acad. Sci. Hungar. 25 (1974),

7. A. Suliński, T. Anderson and N. Divinsky, Lower radical properties for associative and alternative rings, J. London Math. Soc 41 (1966), 417-424.

8. R. Wiegandt, Radical and semisimple classes of rings, Queen's University, Kingston, Ontario, 1974.

INSTITUTE OF MATHEMATICS

UNIVERSITY OF WARSAW, BialYSTOK Division

Akademicka 2, 15-267 Bialystok

POLAND
INSTITUTE OF MATHEMATICS

UNIVERSITY OF WARSAW

Pkin, 00-901 Warsaw

Poland 